\title{
El rol de las redes sociales virtuales en la difusión de información y conocimiento: estudio de casos
}

The Role of Virtual Social Media in the Diffusion of Knowledge and Information: Case Study

O papel das redes sociais virtuais na difusão de informação e conhecimento: estudo de casos

\author{
Marisa Analia Sánchez \\ Giselle Pinochet Sánchez
}

Fecha de recibido: 3 de mayo de 2016

Fecha de aprobado: 14 de julio de 2016

Doi: http://dx.doi.org/10.12804/http://revistas.urosario.edu.co/index.php/empresa/

article/view/4847

Para citar: Sánchez, M. A., \& Pinochet Sánchez, G. (2017). El rol de las redes sociales virtuales en la difusión de información y conocimiento: estudio de casos. Universidad \& Empresa, 19/32/, 107-135.Doi: http://dx. doi. org/10.12804/http://revistas.urosario.edu.co/index.php/empresa/article/view/4847

\section{RESUMEN}

El objetivo del trabajo es analizar la utilización de las redes sociales virtuales en organizaciones de servicios y cómo pueden estas servir de apoyo para la difusión de la información y el conocimiento. La metodología de la investigación se basa en el estudio de casos múltiples e incluye una revisión de la literatura, la realización de cuestionarios con integrantes de organizaciones que se desempeñan en el área de servicios y el análisis de redes sociales elaboradas a partir de la Fan Page de Facebook y los perfiles de LinkedIn. Como resultado del análisis

\footnotetext{
Doctora en Ciencias de la Computación de la Universidad Nacional del Sur (Argentina). Profesora Asociada a tiempo completo del Departamento de Ciencias de la Administración, de la Universidad Nacional del Sur (Bahía Blanca, Argentina). Correo electrónico: mas@uns.edu.ar

** Psicóloga de la Universidad Nacional de Colombia. Especialista en Gerencia de Recursos Humanos de la Universidad Jorge Tadeo Lozano. PhD (c) Ciencia Aplicada de la Universidad Antonio Nariño (Bogotá, Colombia). Correo electrónico: gpinochet@uan.edu.co
} 
de casos se observa un limitado uso de las redes sociales. Así, Facebook registra una actividad frecuente para el caso de organizaciones gubernamentales y académicas; LinkedIn es utilizado a nivel personal, pero no institucional. Finalmente, excepto para un caso vinculado también con el sector productivo, aquellos que utilizan LinkedIn no evidencian que la red constituya un canal importante de transferencia y absorción de información y no muestran convencimiento con respecto a la contribución de las redes sociales en el éxito futuro de la organización.

Palabras clave: Redes sociales virtuales, gestión del conocimiento, intercambio de conocimiento, LinkedIn, Facebook, Colombia.

\section{ABSTRACT}

The objective of this paper is to analyze the usage of virtual social media in organizations in the service area and how these can help to spread information and knowledge. The methodology of the investigation is based on the study of multiple cases and includes a revision of literature, the realization of surveys with members of organizations in the service area, the analysis of social media through Fan pages of Facebooks and profiles in LinkedIn. As a result, the analysis showed a limited usage of social media is noted. Therefore, Facebook registers a frequent activity in the case of government and academic organizations. LinkedIn is used in personal level, but not institutional. Finally, with the exception of a particular case in the production sector, those who utilize LinkedIn do not evidence that the network constitutes an important channel of transference and absorption of information and do not show confidence about the contribution of social media in the future success of the organization.

Keywords: virtual social media, knowledge management, knowledge exchange, LinkedIn, Facebook, Colombia.

\section{RESUMO}

O objetivo do trabalho é analisar a utilização das redes sociais virtuais em organizações de serviços e como podem estas servir de apoio para a difusão da informação e o conhecimento. A metodologia da investigação baseia-se no estudo de casos múltiplos e inclui uma revisão da literatura, a realização de questionários com integrantes de organizações que se desempenham na área de serviços, a análise de redes sociais elaboradas a partir da Fan Page de Facebook e os perfis de LinkedIn. Como resultado da análise de casos observase um limitado uso das redes sociais. Assim, Facebook registra uma atividade frequente para o caso de organizações governamentais e acadêmicas; LinkedIn é utilizado ao nível pessoal, mas não institucional. Finalmente, a exceção de um caso vinculado também com o setor produtivo, aqueles que utilizam LinkedIn não evidenciam que a rede constitua um canal importante de transferência e absorção de informação e não mostram convencimento com respeito à contribuição das redes sociais no sucesso futuro da organização.

Palavras-chave: redes sociais virtuais, gestão do conhecimento, intercâmbio de conhecimento, LinkedIn, Facebook, Colômbia. 


\section{INTRODUCCIÓN}

La comunicación a través de redes sociales virtuales, como Facebook, LinkedIn, Twitter, MySpace y los blogs, entre otras, se ha convertido en uno de los principales medios para estar conectados con amigos, empleados y colegas. Estas ofrecen un medio económico, ubicuo y masivo, y han generado muchas expectativas debido al potencial para dar apoyo a diferentes funciones de una organización. Las posibilidades de comunicación basadas en los medios sociales facilitan el trabajo colaborativo. Muchas organizaciones implementan plataformas de medios sociales, como sistemas de gestión del conocimiento para incrementar el conocimiento compartido (Ellison Gibbs \& Weber, 2005). En particular, las redes sociales ayudan en la difusión de información y conocimiento haciéndola más eficiente. De cualquier manera, existe evidencia de que las organizaciones no siempre obtienen los beneficios esperados.

El modelo de la Triple Hélice resalta la importancia de la sinergia entre los sectores académico, gubernamental y productivo; esto con el objetivo de generar nuevos formatos institucionales y sociales para la producción, transferencia y aplicación de conocimiento (Etzkowitz H., 2003; Etzkowitz \& Leydesddorff, 1995;
Fuerlinger, Fandl \& Funke, 2015). En particular, la importancia de considerar a las universidades en la difusión de conocimiento, ha sido tratada por diversos autores. Bueno (2007), por ejemplo, describe el concepto de tercera misión de la universidad, que aborda cuestiones referidas a la necesidad de aplicar y transferir el conocimiento adquirido en una casa de estudios para dar respuesta a las demandas sociales y concretar la función emprendedora y de innovación. Esto como un compromiso de la universidad en tanto agente de creación y transferencia de conocimiento al interior de la sociedad actual; asimismo, los Gobiernos nacionales, provinciales y municipales utilizan las redes sociales como parte de programas de Gobierno electrónico.

En Colombia, los objetivos estratégicos en materia social, económica y ambiental incluyen el desarrollo empresarial, el cual pretende impulsar la micro, pequeña y mediana empresa, así como el desarrollo productivo del país. Dentro de las necesidades identificadas para alcanzar estas metas están la inserción en la economía global, el aumento de la productividad y la disminución de la informalidad. Para esto se lleva a cabo una estrategia de competitividad, que incluye una política de apoyo a micro, pequeñas y medianas empresas (MIPyMES) en materia 
de formalización y de capital humano, entre otras cosas. De manera complementaria, la iniciativa de masificar el uso de Internet en este tipo de organizaciones obedece también a esos objetivos. Datos del Ministerio de Tecnologías de la Información y las Comunicaciones revelaron que la cobertura de Internet entre las MIPyMES fue del $74 \%$ en 2014. Estos indicaron, además, que el $41 \%$ de dichas empresas tenía presencia en las redes sociales, en donde Facebook, Twitter, Youtube y LinkedIn poseían una penetración del $62 \%, 18 \%, 10 \%$ y $4 \%$, respectivamente (Ministerio de Tecnologías de la Información y las Comunicaciones, 2015).

Ahora bien, de acuerdo con información del Departamento Administrativo Nacional de Estadística de Colombia(DANE), la mayoría de las microempresas colombianas aún no usan Internet para impulsar sus negocios. Según datos de 2015, el 26,2\% de los microestablecimientos encuestados tiene acceso o usa Internet. El porcentaje de personal que lo utiliza asciende a 22,9\%. Además, de acuerdo con esta fuente, solo el $5,9 \%$ tiene página web y únicamente el $4 \%$ está presente en las redes sociales (DANE, 2015).

Considerando el impacto potencial de las redes sociales en la difusión de información y conocimiento, se plantea el objetivo general de este trabajo. El mismo se describe, específicamente, como analizar el rol de las redes sociales virtuales en la difusión de información y conocimiento. Para alcanzar este fin, se optó por una metodología de estudio de caso múltiple, en relación con la utilización de redes sociales en ocho organizaciones que se desempeñan en el área de servicios en la ciudad de Bogotá, Colombia. Con este estudio se pretende avanzar en el conocimiento del problema y destacar la importancia del tema.

El trabajo se organiza de la siguiente forma. En la primera sección se introducirán conceptos básicos acerca del análisis de redes sociales, dado que esta es una de las técnicas centrales utilizadas para realizar el presente estudio; además, se describirán brevemente las plataformas de redes sociales virtuales y los principales trabajos relacionados, esto para poner en contexto la presente investigación. La segunda sección estará dedicada a describir los detalles de la metodología. Luego, se presentarán los resultados y su interpretación. Finalmente, en la sección 6 se sintetizarán las conclusiones y plantearán algunas propuestas de trabajo futuro. 


\section{MARCO REFERENCIAL Y TRABAJOS RELACIONADOS}

\subsection{Breve introducción al Análisis de Redes Sociales}

El Análisis de Redes Sociales aborda el tratamiento de redes de dos formas. Una utiliza redes egocéntricas, que proveen una vista desde la perspectiva del actor en la red y, la otra, que se concentra en la estructura completa de la red (Edwards, 2010; Hanneman \& Riddle, 2005; Haythornthwaite, 1996). En el primer caso, los vínculos que los actores individuales mantienen con otros describen su propia red egocéntrica. Estos describen una foto de un actor típico en un ambiente en particular y muestran cuántos vínculos tiene un actor determinado con otro, qué tipo de vínculos mantienen y qué clase de información brindan o reciben de otros actores en la red. Este análisis es útil cuando el tamaño de la población es grande o cuando es difícil definir los límites de la misma.

En el segundo caso, las redes sociocéntricas (o completas) describen los vínculos que todos los miembros de un ambiente mantienen con el resto de los miembros del ambiente. En principio, este enfoque requiere respuestas de todos los miembros sobre el resto de los miembros. Este requerimiento limita el tamaño de las redes que es posible examinar.
Los datos relacionales que se recopilan en un estudio se representan con grafos. Los actores se corresponden con vértices en el grafo y las relaciones se representan con arcos entre los nodos. En los grafos dirigidos, un arco es un par ordenado. Este último representa la dirección del arco que vincula dos vértices. En los grafos no dirigidos, no existe una dirección asociada al arco.

La posición de un nodo dentro de su red permite inferir su importancia en el intercambio de información y conocimiento. Se utilizan diferentes indicadores basados en los vértices. El grado está dado por la cantidad de arcos que inciden en un vértice. Los vértices que están incluidos en muchos de los caminos más cortos entre otros vértices poseen la Centralidad de Intermediación más alta y tienen la capacidad de conectar dos nodos que, de otra forma, no se vincularían.

Un indicador global del grafo está dado por la densidad del mismo. Esta indica cuán interconectados están los vértices entre sí. Una red con una densidad del $100 \%$ se lograría si todos los nodos estuvieran interconectados. Algunos estudios indican que una estructura poco densa (o no redundante) favorece el flujo de información y conocimiento dentro de la red. Por otro lado, la densidad puede 
ser clave para la colaboración, dado que facilita la construcción de confianza.

\subsection{Plataformas de redes sociales}

La era moderna de las redes sociales comenzó con la mejora en el desempeño de Internet, a partir de 1995. En el periodo 2002-2004 aparecieron y se promocionaron, en particular, redes como Cyworld, Friendster, Plaxo, Reunion.com, Hi5, LinkedIn, MySpace, Orkut, Facebook y Live Spaces (Skeels \& Grudin, 2009). Facebook comenzó como un sitio orientado a los jóvenes en ambientes universitarios y actualmente es utilizado para compartir fotos o vínculos con amigos o publicar mensajes en la "biografía" de otra persona. Dada la próspera tendencia de la utilización de Facebook, muchas organizaciones se han convertido en unos de los principales usuarios para sus estrategias de marketing (Narayanan et al., 2012). Las empresas pagan pospromocionales, utilizan servicios de publicidad o crean una "Fan page" en forma gratuita. Los post de las Fan pages se difunden al público en general y a los usuarios que se suscriben a esta, seleccionando el ícono "Me gusta" en la misma (Shin, Lee, \& Hall, 2014). LinkedIn se enfoca en la información profesional y estimula a sus usuarios para que construyan un currículum vitae abreviado y establezcan conexiones. LinkedIn brinda soporte para la creación de grupos, a través de una aplicación y un proceso de aceptación. Los grupos incluyen redes de alumnos, empleados de una empresa, una organización profesional o grupo de interés.

\subsection{Transferencia y difusión de información y conocimiento}

La transferencia de conocimiento en las organizaciones es el proceso a través del cual unaunidad (porejemplo, un grupo, departamento, o división) es afectado por la experiencia de otro (Argote \& Ingram, 2000). Tal como observan Argote e Ingram (2000), si bien la transferencia de conocimiento en las organizaciones involucra la realización de este proceso a nivel individual, el problema de llevarlo a nivel organizacional trasciende lo individual para incluir, justamente, transferencia a niveles superiores, comogrupos, líneas de productos, departamentos o divisiones.

Dado que la transferencia de conocimiento impacta en el desempeño de las unidades receptoras, esta puede evaluarse midiendo cambios en el conocimiento o en el desempeño (Darr, Argote \& Epple, 1995). Los enfoques basados en este último presentan algunos desafíos, como el control de factores diferentes a la experiencia 
de otras unidades que puedan afectar el desempeño de la unidad receptora. Los enfoques basados en conocimiento resultan más difíciles de instrumentar, debido a que parte del conocimiento puede ser tácito $\mathrm{y}$ no puede capturarse mediante un reporte verbal. Además, es posible que surjan dificultades para medir cambios de conocimiento en diferentes repositorios (por ejemplo, individuos, estándares, prácticas) (Argote \& Ingram, 2000).
La literatura vinculada con la trasferencia o difusión del conocimiento ha tenido un crecimiento sostenido en coincidencia con el florecimiento de los temas de gestión del conocimiento. La figura 1 evidencia este hecho, mostrando la cantidad de artículos recuperados en una búsqueda en Scopus para los términos knowledge management, knowledge transfer y knowledge diffusion, incluyendo un periodo que va hasta finales del mes de marzo de 2016.

Figura 1. Cantidad de documentos recuperados de Scopus para tres consultas que incluyen las expresiones knowledge management, knowledge transfer o knowledge diffusion

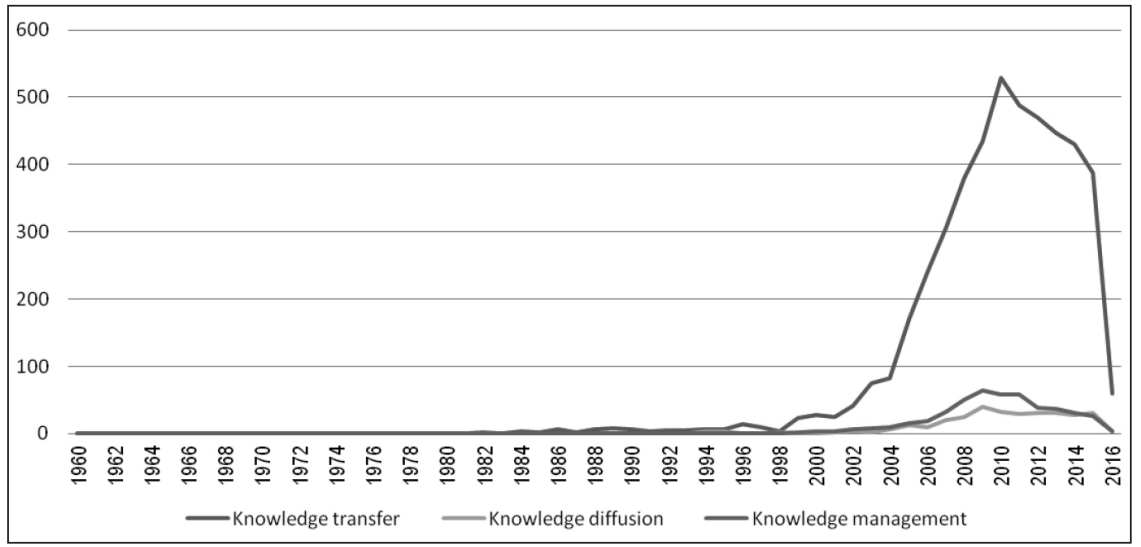

Fuente: elaboración propia, a partir de datos recopilados de Scopus.

Los trabajos sobre difusión de conocimiento cubren un amplio espectro de tópicos de investigación (Luo, Du, Liu, Xuan \& Wang, 2015). El foco del presente estudio es analizar cómo se difunde el conocimiento en las redes sociales. Si bien existen nu- merosos trabajos empíricos yrevisiones, destacamos el trabajo de Phelps, Heidl y Wadhwa (2012), quienes realizan una revisión de cuatro décadas de trabajos que abordan la influencia de las redes sociales en los procesos de creación, difusión, 
absorción y utilización de conocimiento. Ellos definen "red de conocimiento" como un conjunto de nodos interconectados por relaciones que habilitan y restringen la adquisición, transferencia y creación de conocimiento que pueden representar elementos tales como repositorios de conocimientos distribuidos, y agentes que buscan, transmiten o crean conocimiento.

Phelps et al. (2012) estructuran su revisión en tres componentes, a saber: 1) el elemento de la red del conocimiento (por ejemplo, propiedades estructurales, relacional, nodales y de conocimiento), 2) el resultado (creación, transferencia y adopción), y 3) el nivel de análisis (interpersonal, intraorganizacional e interorganizacional). El trabajo concluye observando que existen tanto puntos de coherencia como de conflicto en los argumentos teóricos y los resultados empíricos. Por ejemplo, no es posible afirmar que una estructura de red particular sea universalmente beneficiosa, dado que el efecto de la densidad puede depender de la tarea de conocimiento realizada, en donde los huecos estructurales son beneficiosos para algunas tareas y la densidad, para otras (Morrison, 2002). Gilsing et al. (2008), por su parte, observan que en la literatura existe un tono poco realista sobre la optimalidad de la estructura de una red que se abstrae de cualquier tipo de contexto. Estos autores estudian redes entre organizaciones con referencia al problema de creación de innovación y consideran propie-dades estructurales y relacionales de las redes.

Algunos otros investigadores han estudiado si los beneficios de los vínculos sociales se extienden a las redes virtuales. Si bien Internet ofrece la oportunidad de conectar individuos que de otra forma no hubieran hecho, Van Alstyne y Brynjolfsson (2005) observan que la tecnología tiene también el potencial de fragmentar comunidades. Por ejemplo, los usuarios pueden utilizar filtros avanzados para ubicar a otros individuos con los mismos intereses e interactuar solo con ellos. A partir de esta observación, varios académicos han investigado la utilización de foros virtuales para compartir conocimiento. Por ejemplo, Hwang, Singh y Argote $(2012,2015)$ estudiaron si los empleados transfieren conocimiento dentro o fuera de los límites y cómo las tendencias cambian como función de la experiencia del proveedor de conocimiento en foros virtuales. Ellos consideran límites dados por la geografía, el estatus y la experiencia y analizan los mensajes en un foro de una empresa con más de 118000 empleados ubicados en diferentes continentes. Los autores concluyen que los tra- 
bajadores transfieren conocimiento con mayor frecuencia con los similares. A medida que los empleados ganan más experiencia en compartir conocimiento en el foro virtual, ponen menos atención a similitudes dadas por la geografía y el estatus, $y$ valoran la similitud definida por la experiencia.

Archambault y Grudin (2012) consideraron las redes Facebook, LinkedIn y Twitter para describir cómo estas se utilizan y si se consideran útiles para la comunicación organizacional y la recuperación. El estudio se basa en una muestra tomada de empleados de Microsoft, e incluye un período de cuatro años, desde 2008 al 2011. En 2008 los empleados encuestados tenían un perfil y utilizaban LinkedIn (49\%) y Facebook (46\%). Si bien la tendencia es de mayor utilización para ambas redes, al observar la utilización diaria se observa que muy pocos reportan utilizar LinkedIn. La red profesional LinkedIn resulta más popular en profesionales en el rango de 25 a 40 años, y constituye una agenda que se actualiza sola a medida que los contactos cambian de trabajo (Skeels \& Grudin, 2009).

Gloor, Dorsaz, Fuehres y Vogel(2013), por su parte, comparan el éxito de emprendedores considerando a su conducta en las redes sociales virtua- les. A partir del análisis de un caso de estudio concluyen que la centralidad de los actores se relaciona con el éxito y la proximidad de personas clave. Según Geenhuizen y Nijkamp (2012) la actividad económica global ha incrementado la especialización en la innovación; de esta forma, es más difícil encontrar los mayores componentes del conocimiento necesarios para innovar y resolver un problema en un mismo lugar. Las empresas de alta tecnología no buscan el conocimiento en forma local, sino el mejor conocimiento disponible en su área competitiva (Geenhuizen \& Nijkamp, 2012).

Además, en un estudio sobre comunidades de práctica virtuales se concluye que la integración de redes sociales intensifica el intercambio de conocimiento entre amigos y previene la detención del intercambio (Pan et al., 2015). Para verificar la hipótesis planteada, los autores del trabajo definen tres criterios, a saber: 1) la tasa de nacimiento del intercambio de conocimiento (probabilidad de que una diada que no intercambió información comience a hacerlo), 2) la actividad del intercambio (frecuencia de intercambios entre diadas) y 3) la tasa de mortalidad (probabilidad de que una diada que intercambia información deje de hacerlo). La fuente de datos se define a partir de una 
comunidad virtual y el periodo de investigación incluye una etapa en la cual la plataforma funciona con una tecnología de foro y otra en la que agrega la posibilidad de definir un perfil y una lista de amigos.

Korzynski (2015) examina el rol de las redes sociales virtuales en el compromiso (o motivación) de los empleados y analiza los factores que tienen influencia en la participación de los líderes en las redes. La investigación se instrumentó a partir de encuestas dirigidas a líderes de equipo, gerentes y directores de empresas Fortune 500. Los resultados indican que el compromiso de los empleados está positivamente asociado al tiempo que los líderes pasan en las plataformas de redes internas, así como al código de conducta virtual y la presencia de una cultura organizacional abierta. Además, se confirmó que el entrenamiento sobre cómo utilizar las redes sociales internas es un buen predictor de las horas dedicadas a las redes.

Oostervink et al. (2016), finalmente, examinan la utilización de medios sociales empresariales para compartir conocimiento, considerando si los usuarios comparten información y actúan de acuerdo a las normas y valores vinculados con su profesión o solamente se involucran en activi- dades para colaborar a favor de la productividad organizacional. A partir del estudio de un caso, los resultados muestran que los profesionales enfrentan algunas ambigüedades como resultado de la lógica institucional y la profesional.

\section{METODOLOGÍA}

El método utilizado para la realización de esta investigación corresponde al estudio de casos múltiples (Yin, 1994). La unidad de análisis es la organización, en la cual trabaja 'el contacto', es decir, quien contestó el cuestionario y brindó la información acerca de su red profesional. Para cada organización se recopiló información a partir de contactos profesionales registrados en la red LinkedIn, la actividad en la $\mathrm{Fan} \mathrm{Pa}$ ge de la organización (en los casos en los que esta existe) y un cuestionario; este último permitió indagar sobre aspectos difíciles de inferir a partir de las redes virtuales. De esta forma, se pretendió cotejar las características de la organización, las características relacionales de la red social virtual y la percepción que tienen los contactos sobre el rol de la red en el intercambio de información y conocimiento.

A continuación, se brindan más detalles metodológicos: 


\subsection{Red profesional Linkedln}

Se propuso construir una red surgida de la red de contactos profesionales LinkedIn de un contacto específico que se desempeña en la organización considerada. En el análisis de la red se calculó la densidad del grafo para tener un indicador de cuán conectados están los nodos de la red entre sí. Además, para cada nodo se indicó el grado y la centralidad de intermediación. No se elaboraron otros indicadores, dado que cada red está desarrollada a partir de una sola persona (ego), por lo tanto, los valores de otros indicadores no agregarían información. Para citar un ejemplo de esto, la distancia geodésica máxima siempre sería igual a dos.

A partir de la información pública descrita por cada miembro en LinkedIn se clasificó a cada uno de ellos, de acuerdo con la pertenencia al ámbito académico, gubernamental o productivo (ver tabla 1) y el país de residencia. La clasificación en estos sectores pretendió analizar la capacidad de la red para difundir información entre sectores diferentes y explorar la sinergia entre los mismos. Esto se basa en el modelo de la Triple Hélice. Si bien el modelo original no considera el sector Organización No Gubernamental (ONG) (Leydesdorff, 2012), en este trabajo se lo discrimina a los efectos de no perder información sobre la naturaleza de las organizaciones estudiadas. Asimismo, dado que algunos usuarios de las redes sociales reflejan un desempeño balanceado en dos sectores (académico y productivo), se optó por clasificarlo como "A-P" para denotar su potencial influencia en los sectores académico y productivo.

Además, con el fin de analizar las interacciones entre diferentes actividades económicas, se clasificaron los nodos de la red, considerando la función que desempeñan actualmente, la cual se encuentra publicada en su perfil de la red social LinkedIn. La clasificación se realizó de acuerdo a la Clasificación Industrial Internacional Uniforme de todas las actividades económicas (CIIU) (Naciones Unidas, 2009). Esta constituye la clasificación internacional de referencia de las actividades productivas y aporta cuatro niveles de clasificación (secciones, divisiones, grupos y clases). Luego de realizar un análisis preliminar de los datos, se observó que para muy pocos casos se disponía de datos para determinar la clase. Por lo tanto, a efectos de elaborar clasificaciones comparables, se utilizó el nivel de división para tipificar a los nodos de la red. 
Tabla 1. Sectores utilizados para clasificar a los miembros de la red

\begin{tabular}{|l|l|}
\hline \multicolumn{1}{|c|}{ Sector } & \multicolumn{1}{c|}{ Descripción } \\
\hline Académico & Miembros que trabajan en universidades o institutos científicos. \\
\hline Productivo & Miembros que trabajan en empresas privadas o por cuenta propia. \\
\hline A-P & Sector científico-tecnológico y productivo. Miembros con afiliación en ambos sectores. \\
\hline Gobierno & $\begin{array}{l}\text { Miembros que trabajan para entidades de gobierno (por ejemplo, ministerios, secretarias } \\
\text { o municipios). }\end{array}$ \\
\hline ONG & Miembros que se desempeñan en Organizaciones No Gubernamentales. \\
\hline No informa & $\begin{array}{l}\text { Se utiliza cuando la información publicada en LinkedIn no es suficiente para determi- } \\
\text { nar la clasificación. }\end{array}$ \\
\hline
\end{tabular}

Fuente: elaboración propia.

\subsection{Interacciones en la Fan Page de Facebook}

El análisis de la Fan Page resulta ser un indicador de la importancia que asigna la organización a la gestión de las redes sociales. Por ejemplo, la frecuencia de actualización y el tiempo de respuesta a consultas reflejan este aspecto. Además, es posible describir la conducta de los usuarios, incluyendo cómo se involucran con los posts y cuáles de estos actores resultan ser más promisorios para incrementar la popularidad de una Fan Page. A partir de cada Fan Page de interés, se construye una red en la cual los nodos corresponden con usuarios y se conectan los usuarios que indicaron "Me gusta" o hicieron comentarios sobre el mismo post.

Debido a que los usuarios de Facebook frecuentemente utilizan un nombre de fantasía y, por lo tanto, no es factible efectuar una clasificación automática del perfil (Gobierno, académico, productivo, etc.), se limitó la clasificación a los nodos más promisorios. Los nodos para los cuales se computó el grado y centralidad de intermediación más altos se clasificaron de acuerdo a los perfiles incluidos en la tabla 1 . La clasificación permitió elaborar conclusiones sobre la capacidad de la red para distribuir información rápidamente, vinculando diferentes sectores.

\subsection{Características relacionales}

Para facilitar la interpretación de las redes virtuales y complementar el análisis con propiedades relacionales difíciles de inferir a partir de la estructura de las redes sociales virtuales, se instrumentó un cuestionario estructurado. Este fue validado con la realización de entrevistas 
previas en Argentina. El mismo se envió a los contactos que brindaron información sobre la red LinkedIn.

Se formularon preguntas para caracterizar el flujo de información y conocimiento. Para definir las preguntas se consideró la propuesta de Fritsch y Kauffeld-Monz (2010). Los autores analizan la transferencia de información y conocimiento en una muestra de redes de innovación. Ellos definieron las redes a partir de un cuestionario postal, en el cual a cada actor de la red se le solicitó que mencionara al socio más importante de la red. Si bien el estudio no considera el caso de redes sociales virtuales, se consideró la metodología para adaptarla, con el fin de evaluar la transferencia de información y conocimiento trasladados a los contactos en la red virtual y la absorción de información por ego de la red virtual.

La fuerza de los lazos involucra una combinación de cantidad de tiempo, servicios recíprocos e intensidad emocional (Granovetter, 1973). Dada una red, es difícil cuantificar para cada contacto su antigüedad o la frecuenciadecomunicación. Puesto que se desea estudiar la red en el contexto de la difusión de información y conocimiento, la cantidad de tiempo se aproximó con la frecuencia en que el individuo se enriquece con información, sugerencias o estímulos que sus contactos realizan en la red (para esto se utilizó una escala de Likert de 5 puntos). Con inspiración en el trabajo de Gilsing (2008), los servicios recíprocos se aproximaron con el alcance de los contenidos y se indagó sobre los temas en los que el individuo se informa a través de la red. La confianza mutua se atendió preguntando si el individuo diría que entre los contactos existe honestidad y confianza (usando una escala de Likert de 5 puntos, con las categorías "De ninguna manera" asociado a 1 y "Mucha" asociado a 5). La intensidad emocional se aproximó preguntando acerca del rol de las redes sociales en el éxito de la organización (ver tabla 2).

El cuestionario incluyó otras preguntas para caracterizar el tipo de actividad, la antigüedad de la organización, el tamaño (cantidad de empleados), el mercado geográfico (local, regional, nacional, internacional) y el desempeño general de esta en los últimos tres años (participación en el mercado, ventas, utilidad sobre ventas, productividad, retorno sobre la inversión). Además, se indagó sobre el número de membresías en diferentes tipos de redes (Cámara de comercio, Asociaciones industriales o sectoriales, Grupos de interés, Grupos virtuales de interés). La diversidad de membresías en diferentes redes brinda diferentes puentes con grupos heterogéneos. 
Tabla 2. Resumen de variables e indicadores

\begin{tabular}{|c|c|}
\hline $\begin{array}{l}\text { Flujo de la } \\
\text { información }\end{array}$ & Indicador \\
\hline $\begin{array}{l}\text { Transferencia de } \\
\text { información }\end{array}$ & $\begin{array}{l}\text { Pregunta: “En su opinión, ¿sus contactos de LinkedIn se benefician de la } \\
\text { información o sugerencias que Usted realiza en dicha red social?". Escala de } \\
\text { Likert de } 5 \text { puntos (Nunca, Rara vez, A veces, A menudo, Siempre). }\end{array}$ \\
\hline $\begin{array}{l}\text { Absorción de } \\
\text { información }\end{array}$ & $\begin{array}{l}\text { Pregunta: “UUsted se enriquece de la información, sugerencias o estímulos } \\
\text { que realizan sus contactos en LinkedIn?”. Escala de Likert de } 5 \text { puntos (Nunca, } \\
\text { Rara vez, A veces, A menudo, Siempre). }\end{array}$ \\
\hline \multicolumn{2}{|l|}{ Fuerza de la relación } \\
\hline Cantidad de tiempo & $\begin{array}{l}\text { Frecuencia de acceso a la red. Escala de Likert de } 5 \text { puntos (Nunca, Rara vez, } \\
\text { A veces, A menudo, Siempre). }\end{array}$ \\
\hline Servicios recíprocos & $\begin{array}{l}\text { Pregunta: “USted diría que gracias a la red se beneficia de la siguiente infor- } \\
\text { mación?”. Selección múltiple de temas (Conocimiento científico; Información } \\
\text { técnica y recursos; Know-how de administración/legales; Información de mer- } \\
\text { cado; Recursos financieros; Nunca por ningún motivo; No sabe). }\end{array}$ \\
\hline Confianza mutua & $\begin{array}{l}\text { Pregunta: “Usted diría que entre los contactos existe honestidad y confian- } \\
\text { za?”. Escala de Likert de } 5 \text { puntos con las categorías "De ninguna manera" } \\
=1 \text { y "Mucha" }=5 \text {. }\end{array}$ \\
\hline $\begin{array}{l}\text { Intensidad } \\
\text { emocional }\end{array}$ & $\begin{array}{l}\text { Pregunta: "¿En su organización se considera que las redes sociales (grupos } \\
\text { de interés en Facebook, contactos o grupos en LinkedIn) que facilitan la } \\
\text { colaboración y el conocimiento mutuo con otras organizaciones contribuyen } \\
\text { en el éxito futuro de la organización?". Escala de likert de } 5 \text { puntos ("Total- } \\
\text { mente de acuerdo" }=1 \text {; "En desacuerdo" }=5 \text { ). }\end{array}$ \\
\hline
\end{tabular}

Fuente: elaboración propia.

\section{RESULTADOS}

Se analizaron ocho empresas que brindan servicios, a saber: servicios de diseño gráfico y asesoría en imagen corporativa, marketing, administración de fondos relacionados con seguridad social, protección del adulto mayor mediante programas culturales y ocupacionales, consultoría en temas relacionados con la extracción de petróleo, do- cencia e investigación en educación virtual, y mantenimiento, actualización tecnológica y remodelación de ascensores. La tabla 3 resume características de las organizaciones. En particular, esta ilustra la presencia en las redes sociales Facebook y LinkedIn. Por motivos de confidencialidad, en ella se utilizan nombres ficticios (códigos simples, a manera de pseudónimos) para las organizaciones. 
Tabla 3. Características de las organizaciones consideradas y su presencia en redes sociales

\begin{tabular}{|c|c|c|c|c|c|c|c|}
\hline \multirow[b]{2}{*}{ Organización } & \multirow{2}{*}{$\begin{array}{c}\text { Actividad } \\
\text { económica* }\end{array}$} & \multirow[b]{2}{*}{ Antigüedad } & \multirow[b]{2}{*}{ Tamaño } & \multirow[b]{2}{*}{ Mercado } & \multicolumn{3}{|c|}{ Presencia en redes sociales } \\
\hline & & & & & Facebook & $\begin{array}{c}\text { LinkedIn } \\
\text { (institucional) }\end{array}$ & $\begin{array}{c}\text { LinkedIn } \\
\text { (empleados) }\end{array}$ \\
\hline \multicolumn{8}{|c|}{ Sector Productivo } \\
\hline O-1 & 74 & entre 2 y 10 & $10-49$ & Nacional & - & - & Sí \\
\hline $\mathrm{O}-2$ & 73 & entre 2 y 10 & $10-49$ & Nacional & Sí & - & Sí \\
\hline O-5 & 9 & entre 2 y 10 & $10-49$ & Local & Sí & - & Sí \\
\hline O-7 & 71 & más de 10 & $\begin{array}{c}\text { más de } \\
100\end{array}$ & $\begin{array}{c}\text { Nacional, } \\
\text { internacional }\end{array}$ & Sí & - & Sí \\
\hline \multicolumn{8}{|c|}{ Sector de Gobierno } \\
\hline $\mathrm{O}-3$ & 84 & más de 10 & $\begin{array}{c}\text { más de } \\
100\end{array}$ & Nacional & Sí & - & Sí \\
\hline O-8 & 84 & más de 10 & $\begin{array}{c}\text { más de } \\
100\end{array}$ & Nacional & Sí & - & Sí \\
\hline \multicolumn{8}{|c|}{ Sector académico } \\
\hline O-6 & 85 & más de 10 & $\begin{array}{c}\text { más de } \\
100\end{array}$ & Nacional & Sí & Sí & Sí \\
\hline \multicolumn{8}{|c|}{ Sector ONG } \\
\hline $\mathrm{O}-4$ & 88 & entre 2 y 10 & $10-49$ & Local & Sí & - & Sí \\
\hline
\end{tabular}

* Código según la Clasificación Industrial Internacional Uniforme de todas las actividades económicas (CIIU) (Naciones Unidas, 2009).

Fuente: elaboración propia.

Para cada organización se exploró Internet con el objetivo de determinar si dispone de una Fan Page o Grupo institucional en la red social Facebook. Para todas las organizaciones con presencia en Facebook se realizó un análisis preliminar de la Fan Page para determinar la fecha de inicio, la cantidad de seguidores y la frecuencia de la actividad (publicación de posts). Solo para las organizaciones O-3, O-6 y O-8 se definió una red a partir de dichos posts. Para el resto no se procedió así, dado que la actividad resulta ser nula. A partir de los grafos que representan las redes, se computó la cantidad de vértices, arcos y la densidad. Con respecto a los vértices se calculó el promedio de los "Me gusta" y los comentarios creados, dado que estos tienen un impacto en el flujo de la información. Los datos elaborados se presentan en la tabla 4.

Con respecto a los contactos profesionales se definió una red para cada organización. Se calcularon las métricas basadas en el grafo, como 
cantidad de vértices, arcos y densidad. El número total de vértices se discriminó de acuerdo al sector (académico, productivo, Gobierno, académico-productivo u $\mathrm{ONG}$ ) vinculado al nodo. Además, para cada vértice se computó el grado y la centralidad de intermediación. A efectos de caracterizar los nodos puente (con valores altos para la centralidad de intermediación) se indica con cuál sector están relacionados los nodos que registran los mayores valores para la centralidad de intermediación. En la tabla 5 se organizan todos los datos procesados para una mejor visualización y comparación. Las tablas 7 y 8 resumen las actividades económicas asociadas a cada nodo y su país de residencia.

A cada uno de los contactos que brindó información sobre su red profesional se le suministró un cuestionario estructurado y se obtuvo respuesta para 5 de los 8 casos. Dado que el objetivo no es realizar un análisis cuantitativo o estadístico de las respuestas, sino complementar la información disponible para cada organización, se presentan los resultados completos en la tabla 6.

Tabla 4. Descripción sintética de las Fan Page analizadas

\begin{tabular}{|c|c|c|c|c|c|c|c|c|}
\hline \multirow[b]{2}{*}{$\begin{array}{l}\text { Nombre de } \\
\text { la Fan Page } \\
\text { o grupo }\end{array}$} & \multirow[b]{2}{*}{ Inicio } & \multirow[b]{2}{*}{$\begin{array}{l}\text { Número de } \\
\text { seguidores/ } \\
\text { miembros }\end{array}$} & \multirow[b]{2}{*}{$\begin{array}{l}\text { Frecuencia } \\
\text { de actividad }\end{array}$} & \multicolumn{2}{|c|}{ Métricas de vértices } & \multicolumn{3}{|c|}{ Métricas del grafo } \\
\hline & & & & $\begin{array}{c}\text { Me gusta } \\
\text { creados } \\
\text { promedio }\end{array}$ & $\begin{array}{c}\text { Comentarios } \\
\text { creados } \\
\text { promedio }\end{array}$ & Vértices & Arcos & Densidad \\
\hline \multicolumn{9}{|c|}{ Sector Productivo } \\
\hline O-1 & - & - & - & - & - & - & - & - \\
\hline $\mathrm{O}-2 *$ & 2014 & 9 & Sin actividad & - & - & - & - & - \\
\hline $\mathrm{O}-5^{*}$ & 2011 & 78 & Sin actividad & - & - & - & - & - \\
\hline $\mathrm{O}-7^{*}$ & 2015 & 18 & Sin actividad & - & - & - & - & - \\
\hline \multicolumn{9}{|c|}{ Sector Gobierno } \\
\hline $\mathrm{O}-3^{*}$ & 2012 & 1961 & Diaria & 0,709 & 0,714 & 155 & 2050 & 0,10 \\
\hline $\mathrm{O}-8^{*}$ & 2010 & 2711 & Semanal & 2,46 & 0,03 & 134 & 2187 & 0,14 \\
\hline \multicolumn{9}{|c|}{ Sector Académico } \\
\hline $\mathrm{O}-6 * *$ & 2012 & 16476 & Diaria & 1,35 & 0,71 & 983 & 134436 & 0,14 \\
\hline \multicolumn{9}{|c|}{ Sector ONG } \\
\hline $\mathrm{O}-4 *$ & 2008 & 121 & Sin actividad & - & - & - & - & - \\
\hline
\end{tabular}

: * Período observado del 1/1/2016 al 15/4/2016.

** Período observado del 1/3/2016 al 1/4/2016.

Fuente: elaboración propia. 


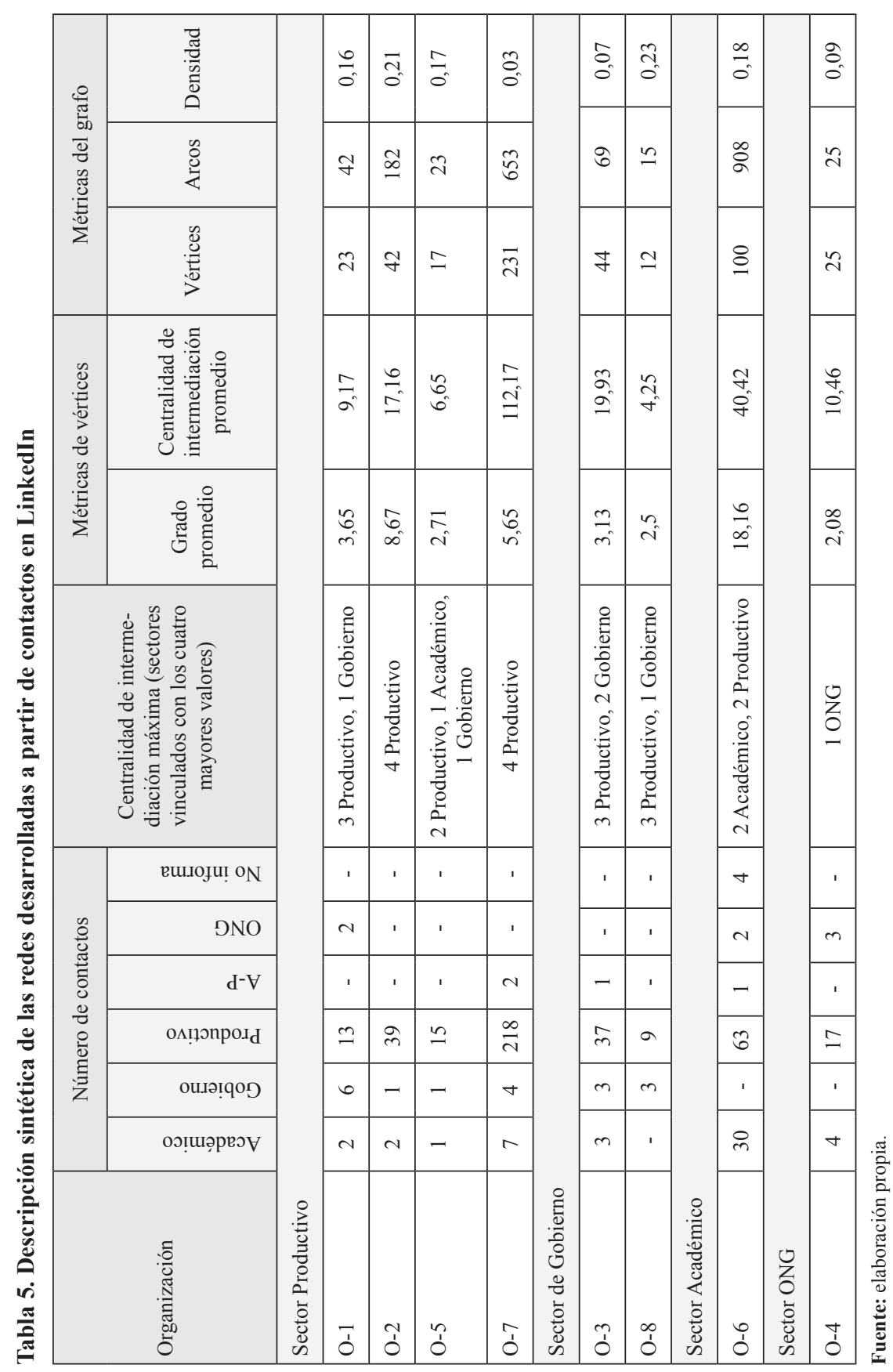




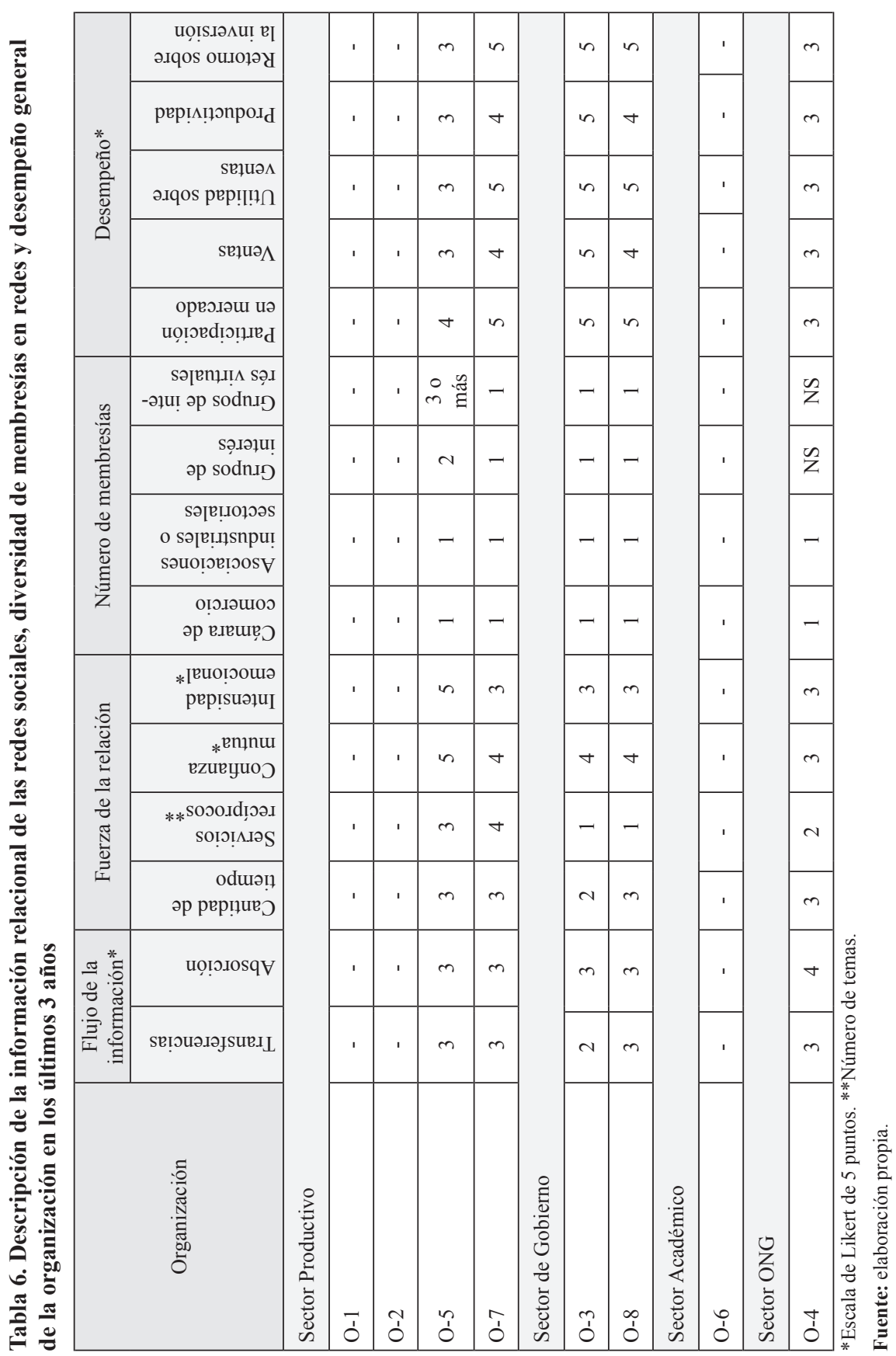




\begin{tabular}{|c|c|c|c|c|c|c|c|c|c|c|c|c|c|c|c|c|c|c|c|c|}
\hline $\begin{array}{l}\infty \\
0 \\
0\end{array}$ & 0 & 0 & 0 & 0 & 0 & 0 & 0 & 0 & + & 0 & 0 & 0 & 0 & $\sim$ & 0 & 0 & $m$ & 0 & 0 & 0 \\
\hline$\hat{0}$ & $\sim$ & 0 & 0 & $\sim$ & $\sim$ & -1 & 0 & $\nabla$ & F & 0 & - & 0 & 0 & $n$ & 0 & 0 & - & 0 & 0 & $\nabla$ \\
\hline \begin{tabular}{l|l}
0 \\
0
\end{tabular} & 0 & 0 & 0 & 0 & $N$ & 0 & - & - & $a$ & $\stackrel{0}{\simeq}$ & 0 & 0 & 0 & 0 & 0 & $\sim$ & 0 & 0 & 0 & 0 \\
\hline 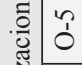 & $\sim$ & 0 & 0 & 0 & 0 & $m$ & 0 & 0 & -1 & 0 & 0 & -1 & 0 & 0 & 0 & 0 & 0 & -1 & 0 & 0 \\
\hline 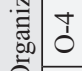 & - & 0 & 0 & 0 & 0 & -1 & 0 & 0 & $\nabla$ & 0 & 0 & 0 & $\sim$ & 0 & 0 & - & 0 & 0 & 0 & 0 \\
\hline$\hat{o}$ & $\sim$ & 0 & 0 & 0 & 0 & 0 & 0 & 0 & -1 & 0 & -1 & 0 & 0 & 0 & 0 & 0 & $\sim$ & $m$ & 0 & - \\
\hline กิ & - & - & - & 0 & 0 & 0 & 0 & 0 & $N$ & 0 & - & 0 & -1 & 0 & - & 0 & - & $\sim$ & -1 & $\sim$ \\
\hline ó & $r$ & 0 & 0 & 0 & 0 & 0 & 0 & 0 & $N$ & 0 & 0 & 0 & -1 & 0 & 0 & 0 & $N$ & 0 & 0 & 0 \\
\hline 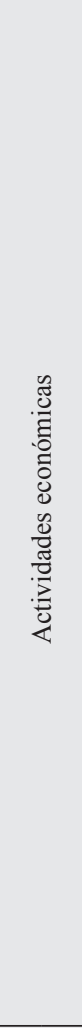 & 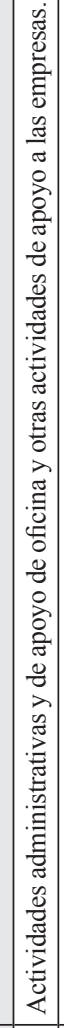 & 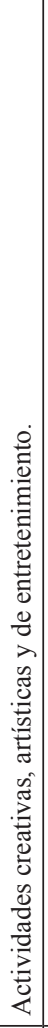 & 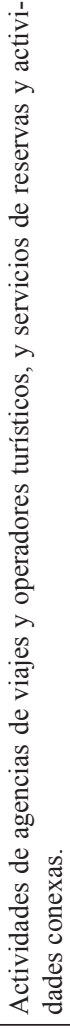 & 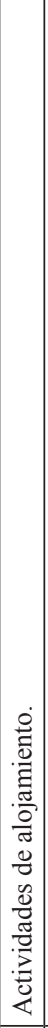 & 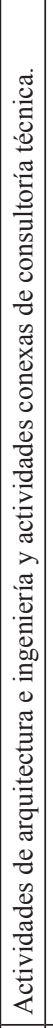 & 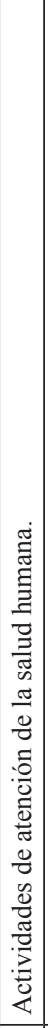 & 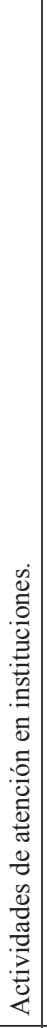 & 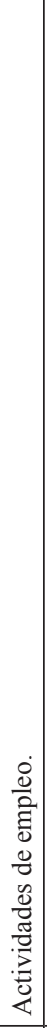 & 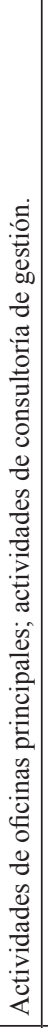 & 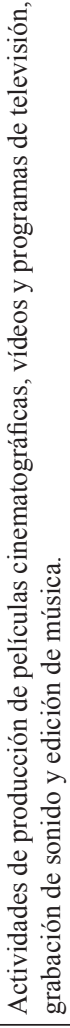 & 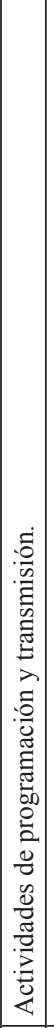 & 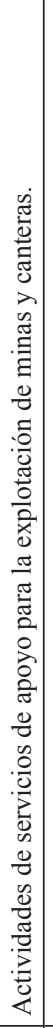 & $\begin{array}{c} \\
\\
\\
\\
\\
\\
0\end{array}$ & 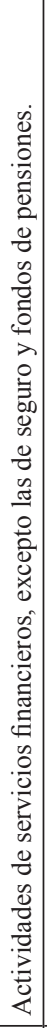 & 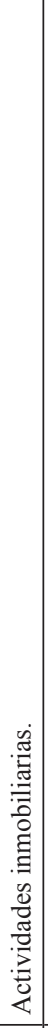 & 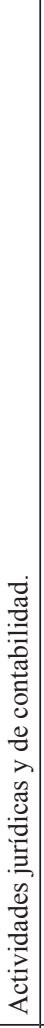 & 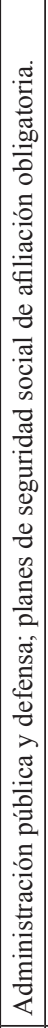 & 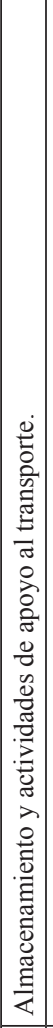 & 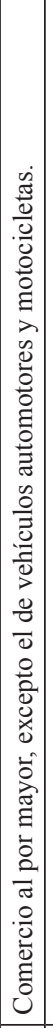 & 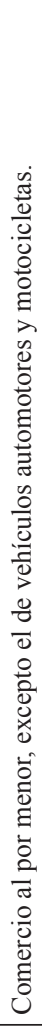 \\
\hline 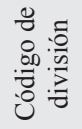 & $\widetilde{\infty}$ & ঃ & $\stackrel{2}{2}$ & $n$ & $\nabla$ & 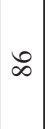 & $\hat{\infty}$ & $\stackrel{\infty}{\sim}$ & $?$ & हे & 8 & a & 3 & t & $\infty$ & bे & ‡ & $\tilde{n}$ & \& & F \\
\hline
\end{tabular}




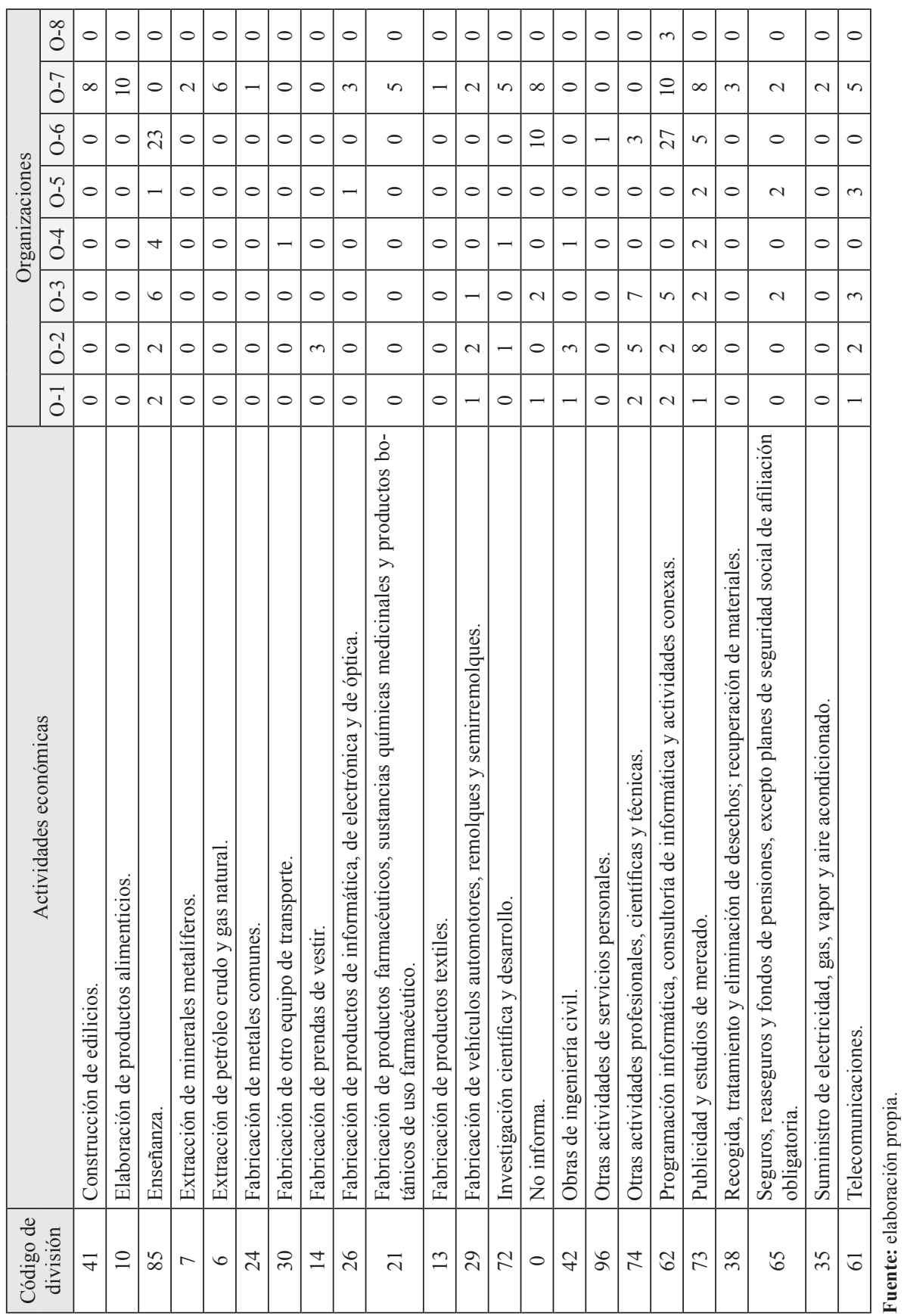


Tabla 8. País de residencia de los miembros incluidos en las redes profesionales para cada organización

\begin{tabular}{|c|c|c|c|c|c|c|c|c|}
\hline & O-1 & $\mathrm{O}-2$ & O-3 & O-4 & O-5 & O-6 & $\mathrm{O}-7$ & O-8 \\
\hline Alemania & - & - & - & - & - & 1 & - & - \\
\hline Argentina & - & - & - & - & - & - & 4 & - \\
\hline Brasil & - & - & - & 1 & - & - & 3 & \\
\hline Canadá & - & - & - & - & - & 1 & & - \\
\hline Chile & 1 & 1 & - & - & - & - & 2 & - \\
\hline China & - & - & - & - & - & - & - & - \\
\hline Colombia & 22 & 22 & 43 & 22 & 17 & 96 & 196 & 11 \\
\hline Costa Rica & - & - & - & - & - & & 3 & \\
\hline Ecuador & - & 2 & - & - & - & - & 2 & - \\
\hline España & - & 1 & 1 & & & 2 & 6 & 1 \\
\hline Estados Unidos & & 1 & - & - & - & - & 3 & \\
\hline India & - & - & - & - & - & - & 2 & - \\
\hline Irlanda & & 1 & & & - & & & - \\
\hline México & - & 3 & & & - & & 4 & \\
\hline Nicaragua & - & 1 & - & - & - & - & - & - \\
\hline No informa & - & - & - & 1 & - & - & - & - \\
\hline Panamá & - & 1 & - & - & - & - & 1 & - \\
\hline Perú & & 1 & - & - & - & - & 2 & - \\
\hline Puerto Rico & & & & & & & 2 & \\
\hline Reino Unido & - & & & & & & 1 & - \\
\hline Venezuela & - & 8 & - & - & - & - & - & - \\
\hline Totales & 23 & 42 & 44 & 24 & 17 & 100 & 231 & 12 \\
\hline
\end{tabular}

Fuente: elaboración propia.

\section{INTERPRETACIÓN DE RESULTADOS}

Dentro del sector productivo se analizaron cuatro organizaciones de las cuales tres cuentan con presencia en Facebook y algunos empleados cuentan con un perfil en LinkedIn. De todos modos, las Fan Page no registran actividad. Asimismo, los perfiles en LinkedIn no revelan más de cincuenta contactos, excepto para el caso de una empresa que brinda servicios de ingeniería (O-7). La red del contacto con afiliación en la empresa O-7 incluye miembros de los sectores académico, productivo y de Gobierno. 
Si se consideran los cuatro mayores valores para la centralidad de intermediación, se observa que corresponden con nodos del sector productivo. Si bien se registran contactos de diversas actividades económicas, la más numerosa corresponde a "Actividades de empleo". Esto último puede estar relacionado con el hecho que la actividad profesional del contacto que brindó la red está vinculada con la gestión de recursos humanos.

Predominan contactos con residencia en Colombia, aunque un $15 \%$ tienen residencia en otros países. $\mathrm{La}$ organización O-7 trabaja en un mercado internacional, por lo tanto, no sorprende la cantidad de contactos que tiene fuera de Colombia. En el cuestionario el contacto opina que "a veces" sus contactos se benefician de la información o sugerencias que realiza en la red social. Tiene una percepción similar con respecto a su enriquecimiento a partir de la información publicada en la red, con lo cual no hay evidencia de que LinkedIn constituya un canal importante de transferencia y absorción de información. Finalmente, el contacto es neutral con respecto a la aseveración sobre la contribución de las redes sociales en el éxito futuro de la organización.

Las redes definidas a partir de contactos en O-1, O-2 y O-5 registran pocos contactos. De todos modos, tal como puede observarse en la tabla 5, los nodos representan diferentes sectores (académico, de Gobierno y productivo). Esto último podría favorecer el flujo de información heterogénea y no redundante. La empresa O-5 contestó el cuestionario y si bien opina que el flujo de la información no es frecuente, la intensidad emocional registra la máxima puntuación. Esto último revela una percepción positiva de las redes sociales por parte de la organización. Para el caso de la empresa O-1, no hay evidencia de la utilización de las redes sociales. No cuenta con una Fan Page en Facebook. Para las organizaciones O-1 y O-5 predominan contactos de Colombia, mientras que casi el $50 \%$ de los contactos de O-2 son de otros países sin predominar ninguno.

Se consideraron dos organizaciones del sector de Gobierno y dedicadas a la prestación de servicios de seguridad social (O-3 y O-8). La actividad en las Fan Page de Facebook es más relevante. Estas constituyen un medio de divulgación de eventos, actividades y respuesta a consultas de ciudadanos. El número promedio de "Me gusta" creados más alto correspondeala FanPageadministrada por O-8, lo cual refleja que los posts publicados resultan de interés para el público. Con referencia a las redes definidas a partir de contactos 
profesionales de miembros de las organizaciones, se observan escasos contactos. La mayoría de los contactos representan al sector productivo. De acuerdo con las respuestas del cuestionario, la transferencia de la información en las redes sociales pro- fesionales solo se produce "a veces" y la fuerza de la relaciones puede definirse como neutral. Las organizaciones registran escasas membresías en otros grupos (no virtuales). Los contactos son mayoritariamente de Colombia.

Figura 2. Red definida a partir de la red social profesional LinkedIn (O-6)

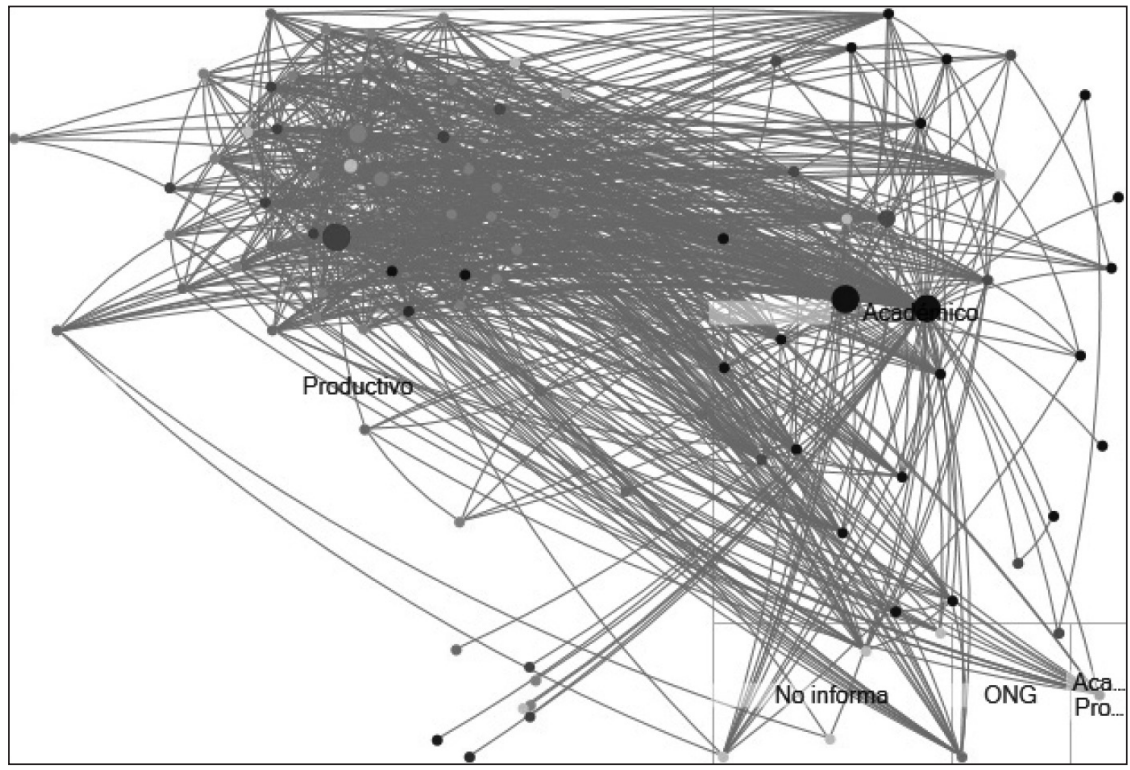

El grupo está definido en base al sector (académico, productivo, A-P, ONG) y el color de los vértices se define de acuerdo a la actividad económica. El tamaño de los vértices se relaciona con la centralidad de intermediación (el tamaño mínimo se mapea con la media del indicador, y el máximo con el segundo valor máximo). Como la red está generada a partir de un contacto, el máximo valor para la centralidad de intermediación corresponde con ese contacto.

Fuente: elaboración propia utilizando el software NodeXL $®$.

Para el sector académico se consideró una universidad identificada como O-6. La misma cuenta con presencia en las redes sociales $\mathrm{Fa}$ cebook y LinkedIn. La actividad en Facebook es diaria. La Fan Page cuenta con 16.476 seguidores y en el periodo observado participaron 983 contactos comentando o indicando "Me gusta" en los posts. Dada la cantidad de post se utilizó una ventana de observación menos extensa que para el resto de los casos. Dicha actividad evidencia transferencia de 
información desde la organización a los lectores de la Fan Page, la cual constituye un medio de vinculación con el público.

El perfil institucional en LinkedIn indica que 16.902 miembros de la red social han indicado que son alumnos o exalumnos. En dicho perfil pueden publicar alumnos, exalumnos o profesores. Se pudo analizar la red profesional definida a partir de los contactos de un empleado y la misma refleja un gran número de contactos de los sectores productivo y académico. No se registran contac- tos en el sector gubernamental (ver figura 2). Si se consideran los cuatro mayores valores para la centralidad de intermediación, se observan nodos del sector académico y productivo. La actividad económica que predomina en los contactos corresponde con la "Enseñanza".

Finalmente, se analizó una ONG (O-4) dedicada a la asistencia social. Si bien cuenta con una Fan Page, la misma no revela actividad. La red profesional construida a partir de un empleado contiene veinticinco contactos, que representan a los sectores

Figura 3. Síntesis de los resultados para las organizaciones que respondieron el cuestionario

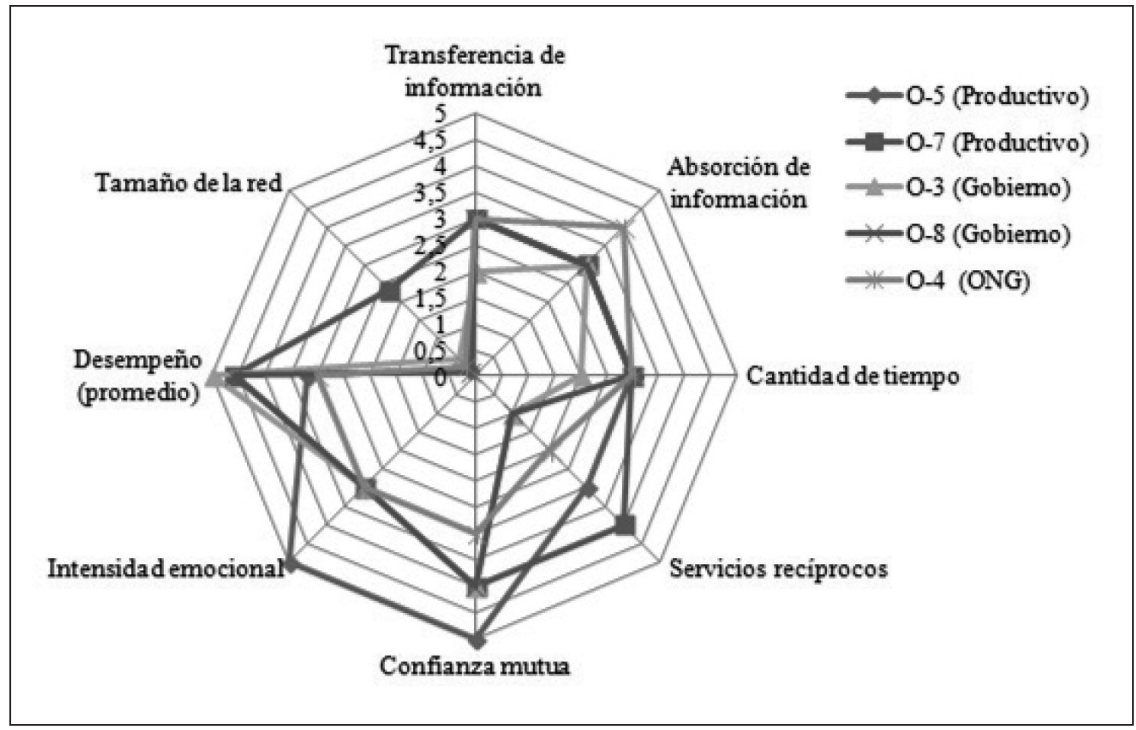

Incluye el tamaño de la red Linked-In, representado como la cantidad de contactos (expresado en centenares). El desempeño promedia los valores registrados para la participación en el mercado, las ventas, la utilidad sobre ventas, la productividad y el retorno sobre la inversión.

Fuente: elaboración propia. 
académico, profesional y ONG. Predominan nodos vinculados con "Actividades administrativas y de apoyo de oficina y otras actividades de apoyo a las empresas". A partir de las respuestas al cuestionario brindadas por el contacto, se observa que sus contactos se benefician "a veces" de la información publicada, mientras que él se beneficia "a menudo". Las respuestas referidas a la fuerza de las relaciones en la red social son neutrales.

En todos los casos, la densidad de la red definida a partir de LinkedIn es baja, siendo el valor máximo 0,23. Esto significa que solo se observa el $23 \%$ de las posibles relaciones entre los nodos de la red. A efectos de la difusión de información y conocimiento, esto puede implicar que los nodos no se conocen entre sí y, por lo tanto, el ego de la red recibe información no redundante $\mathrm{y}$ de diferentes fuentes.

\section{CONCLUSIONES Y TRABAJO FUTURO}

En este trabajo se presenta un estudio de casos múltiple con el objetivo de analizar el rol de las redes sociales virtuales en la difusión de información y conocimiento. Se tomó como unidad de análisis a organizaciones que utilizan redes sociales y se desempeñan en el sector académico, gubernamental, productivo y las ONG. En el análisis de redes sociales se consideraron Facebook y LinkedIn. El análisis de las redes que surgen a partir de LinkedIn incluye no solo los indicadores basados en el grafo y los vértices, sino que también se realizó una clasificación de cada nodo, considerando la actividad económica asociada a la organización en la cual trabaja y su país de residencia.

Si bien muchos autores destacan la importancia de las redes sociales como medio de difusión de información, en la mayoría de las organizaciones estudiadas no se evidencia la misma percepción. Facebook parece ser un canal relevante para tres organizaciones, dos del sector gubernamental y otra del académico (O-3, O-8 y O-6). Estas empresas aprovechan esta red social como medio de vinculación con el público. Por otro lado, la presencia en LinkedIn a nivel institucional es prácticamente nula y no se aprecia que las mismas utilicen un perfil como canal de comunicación o aprovechen el soporte de LinkedIn para la creación de grupos.

Para el caso de una organización del sector productivo (O-7), el contacto analizado cuenta con un perfil LinkedIn con numerosos contactos que se desempeñan en diversas 
actividades económicas y que residen tanto en Colombia como en el resto del mundo. Sin embargo, a partir del cuestionario, el contacto no sugiere que LinkedIn constituya un canal importante de transferencia y absorción de información y no refleja convencimiento con respecto a la contribución de las redes sociales en el éxito futuro de la organización.

A partir del análisis de los casos, solo un contacto -que se desempeña en el sector productivo y presta servicios de apoyo para la explotación de minas y canteras (O-5)- es más positivo con respecto al rol de las redes sociales en la difusión de información y como factor que contribuye al éxito de las organización. De cualquier manera, la red profesional del contacto tiene menos de treinta conexiones y la organización no posee una Fan Page.

En suma, si se considera integralmente el análisis de las redes que surgen de LinkedIn y Facebook, y los cuestionarios, no existe evidencia de que las organizaciones consideren la utilización de las redes sociales como una estrategia relevante. Solo se destaca la utilización de las Fan Page por parte de las organizaciones gubernamentales.

De este modo, el estudio contribuye a entender cómo las organizaciones hacen uso y se benefician de la utilización de las redes sociales para dar apoyo a actividades de transferencia y absorción de información. Como trabajo futuro se pretende ampliar el estudio para considerar una muestra de casos que permita aplicar una metodología cuantitativa para obtener conclusiones de carácter más general.

\section{REFERENCIAS}

Archambault, A., \& Grudin, J. (2012). A Longitudinal Study of Facebook, Linkedin, and Twitter Use. En R. Grinter, Proceedings of the SIGCHI Conference on Human Factors in Computing Systems, 2741-2750. Austin: ACM.

Argote, 1., \& Ingram, P. (2000). Knowledge Transfer: A Basis for Competitive Advantage in Firms. Organizational behavior and Human Decision Processes, 82(1), 150-169.

DANE. (2015). Indicadores básicos de tenencia y uso de TIC en microestablecimientos. Bogotá: DANE.

DANE. (2015). Indicadores básicos de TIC en empresas. Bogotá: DANE.

Darr, E., Argote, L., \& Epple, D. (1995). The adquisition, transfer and depreciation of knowledge in servece organizations: Pro- 
ductivity in franchises. Management Science, 41, 1750-1762.

Edwards, G. (2010). Mixed-Method Approaches to Social Network Analysis. CIUDAD: ESRC National Centre for Research Methods.

Ellison, N., Gibbs, J., \& Weber, M. (2015). The use of enterprise social networks sites for lnowledge sharing in distributed organization: the roles of organizational affordances. American behavioral Scientist, 29(1), 103-123.

Etzkowitz, H. (2003). Innovation in Innovation: The Triple Helix of University-Industry-Government Relations. Social Science Information, 42(3), 293-337.

Etzkowitz, H., \& Leydesddorff, L. (1995). The Triple Helix -University-Industry-Government Relations: A Laboratory for Knowledge Based Economics Development. EASST Review, 14(1), 11-19.

Fritsch, M., \& Kauffeld-Monz, M. (2010). The impact of network structure on oknowledge transfer: an application of social network analysis in the context of regionanl innovation networks. The Annals of Regional Science, 44, 21-38.

Fuerlinger, G., Fandl, U., \& Funke, T. (2015). The role of the state in the entrepreneurship ecosystem. Insights from Germany. Triple Helix, 2(3), 1-26.
Geenhuizen, M., \& Nijkamp, P. (2012). Knowledge virtualization and local connectedness among young globalized high-tech companies. Technological Forecasting \& Social Change, 79, 11791191.

Gilsing, V., \& Duysters, G. (2008). Understanding novelty creation in exploration networks -Structural and relational embeddedness jointly considered. Technovation, 28, 693-708.

Gloor, P., Dorsaz, P., Fuehres, H., \& Vogel, M. (2013). Choosing the right friends -predicting success of startup entrepreneurs and innovators through their online social network structure. Int. $J$. Organisational Design and Engineering, 3(1), 67-85.

Granovetter, M. (1973). The strength if weak ties. American Journal of Sociology, 78, 1360-1389. Hanneman, R., \& Riddle, M. (2005). Introduction to social network methods. Riverside: University of California.

Haythornthwaite, C. (1996). Social Network analysis: An Approach and Technique for the Study of Information Exchange. Library \& Information Science Research, 18(4), 323-342.

Hwang, E., Singh, P., \& Argote, L. (1 de 2012). Learning to Cross Boundaries in Online Knowledge Communities: Fading of Surface-level and Rise of Deep-level 
Similarity with Experience. Carnegie Mellon University. Research Showcase @ CMU.

Hwang, E., Singh, P., \& Argote, L. (2015). Knowledge Sharing in Online Communities: Learning to Cross Geographic and Hierarchical Boundaries. Organization Science, 26(6), 1593-1611.

Korzynski, P. (2015). Online networking and employee engagement: What current leaders do? Journal of Management Psychology, 30, 582-596.

Leydesdorff, L. (2012). The Triple Helix, Quadruple Helix, ..., and an N-Tuple of Helices: Explanatory Models for Analyzing the Knowledge-Based Economy? Journal of Knowledge and Economics, 3, 25-35.

Luo, S., Du, Y., Liu, P., Xuan, Z., \& Wang, Y. (2015). A study on coevolutionary dynamics of knowledge diffusion and social network structure. Expert Systems with Applications, 42, 3619-3633.

Ministerio de Tecnologías de la Información y las Comunicaciones. (10 de 7 de 2015). Caracterización de las MIPyMEs Colombianas en relación con las TIC. Recuperado de http:// www.mintic.gov.co/portal/604/ w3-article-11147.html

Morrison, E. (2002). Newcomers' relationship: the role of social network ties during socialization. Academy of Mangement Journal, 45, 1149-1160.

Naciones Unidas. (2009). Clasificación Industrial Internacional Uniforme de todas las actividades económicas (CIIU). Revisión 4. Departamento de Asuntos Económicos y Sociales. Nueva York: Naciones Unidas.

Narayanan, M., Asur, S., Nair, A., Rao, S., Kaushik, A., Mehta, D., ... Lalwani, R. (2012). Social Media and Business. Vikalpa: The journal for Decision Makers, 37(4), 69-111.

Oostervink, N., Agterberg, M., \& Huysman, M. (2016). Knowledge Sharing on Enterprise Social Media: Practices to Cope with Institutional Complexity. Journal of Computer-Mediated Communication, 21, 156-176.

Pan, Y., Xu, Y., Wang, X., Zhang, C., Ling, H., \& Lin, J. (2015). Integrating social networking support for dyadic knowledge exchange: a study in a virtual community of practice. Information \& Management, 52, 61-70.

Phelps, C., Heidl, R., \& Wadhwa, A. (2012). Knowledge, Networks, and Knowledge Networks: A Review and Research Agenda. Journal of Management, 38(4), 1115-1166.

Shin, S., Lee, K., \& Hall, D. (2014). exploring Facebook Users' 
Continuous Visiting Behaviors: Conceptual Incorporation of Facebook User Perceptions toward Companies'Facebook Fan Page Usage. Twentieth Americas Conference on Information Systems. Savannah.

Skeels, M., \& Grudin, J. (2009). When social networks cross boundaries: a case study of workplace use of Facebook and Linkedin. Proceedings of the
ACM 2009 International Conference on Supporting Group Work (págs. 95-104). Florida: ACM. Van Alstyne, M., \& Brynjolfsson, E. (2005). Global village or CyberBalkans: Modeling and measuring the integration of electronic communities. Management Science, 51(6), 851-868.

Yin, R. (1994). Case Study Research: Design and Methods. Thousand Oaks: Sage Publications. 